\title{
Semántica del entorno educativo. La representación subjetiva del espacio
}

\author{
Semantyc of the educational environment. The subjective \\ representation of space
}

Javier Herrero-Martín

\section{Introducción}

E espacio en el que vivimos es un factor esencial en el análisis y en el comportamiento de los seres humanos (Poldma, 2010). La forma en que las personas se desenvuelven en el territorio es un reflejo relevante de las propiedades del espacio, del resultado de la interacción entre la propuesta formal del mismo y la manera en que los individuos responden cognitiva, afectiva, motriz y socialmente a ella (Davies et al., 2010).

El espacio escolar no es un mero actor pasivo del proceso de aprendizaje. Por ello, su análisis compromete la acción educadora (Laorden y Pérez, 2002). Las personas, adultos y niños responden de forma discriminada ante diferentes propuestas de organización (Kenkmann, 2011). Así, los niños transitan a lo largo y ancho de la escuela Infantil y Primaria, por escenarios muy diversos que modifican su modo de hacer y, muy probablemente con ello, su modo de pensar. Por esta razón, la "competencia para la organización del entorno" de los maestros y educadores (environmental competence, Lackney, 2008) es un argumento a tener en cuenta a la hora de planificar su formación, pues ocurre que, de lo contrario, este analfabetismo espacial (e incluso temporal) impacta en el desarrollo de nuevas propuestas de diseño, los Ilamados NGLE's, o new generation learning environments (Cleveland y Fisher, 2014; Imms, Cleveland and Fisher, 2016).

La investigación general sugiere la existencia de una diversidad de factores en la determinación del uso del espacio educativo. Algunas experiencias desafían los modelos más tradicionales en educación. En ellas, los espacios que dialogan con las personas representan un sistema en permanente cambio (por ejemplo, el diseño de aula de aprendizaje activo, ALC; Park y Choi, 2014). A diferencia del espacio entendido como algo estático, físico, estable, la relación entre subjetividad y entorno supone la construcción de narrativas diferentes, en las que el movimiento, la acción y la representación del niño y del adulto, se confunden con las modificaciones en el ambiente, con la utilización diversa de los espacios o con la creación, reconstrucción o transformación de nuevas y variadas propuestas lógicas (Gislason, 2007). El espacio físico, formal, es considerado como un mero contenedor de la actividad, cuyas señas de identidad permanecen ancladas en visiones tradicionales, conservadoras y alejadas de brillos innovadores. Sin embargo, la inclusión de la persona en el sistema añade una nueva dimensión, el especio mental o espacio 
representado, que transciende la capa física y va más allá de su extensión formal.

La arquitectura y el interiorismo se han ocupado tradicionalmente de analizar las condiciones en las que el espacio puede ofrecer nuevas y enriquecedoras posibilidades de expansión a las personas. Curiosamente, no tanto, la investigación refleja lo propio en el ámbito educativo. El espacio físico pierde identidad si no se considera el espacio mental y el espacio social como parte de un todo integrado (Lefebvre, 1991; Perolini, 2011). Su naturaleza, su grado de adaptación al individuo o de estimulación del aprendizaje, son condiciones que deben ser analizadas en la búsqueda de mejorar los resultados en la escuela y en las instituciones educativas (Barret, y Zhang, 2009; Barrett, Davies, Zhang, y Barrett, 2015).

El objeto del presente estudio es considerar las relaciones entre la representación subjetiva del usuario y la representación física del espacio, analizando su impacto sobre la utilización del espacio educativo y la atribución de funciones diversas de entornos de aprendizaje. Dicho impacto se considera un factor crítico determinante en las nuevas propuestas de innovación educativa que posibiliten una transformación dotada de un componente semánticotransformacional que garantice el éxito de los proyectos.

\section{La semántica del espacio}

El concepto de espacio se entiende como una abstracción, como una representación de un continente en un sistema en el que la relación del individuo (o los individuos), con el entorno de actuación otorgan modelos o patrones de identidad característicos a cada situación dada. Esta representación puede analizarse en relación a un conjunto limitado de planos superpuestos:

a) la representación física. El espacio tal y como lo vemos. El espacio percibido, en primera instancia, limitado sensorial y físicamente por nuestras propias capacidades de reconocimiento formal del mundo.

b) la representación funcional. Un espacio se define también, al igual que los objetos, por su funcionalidad. Así, un pasillo es un espacio de tránsito, un hall de entrada, de recepción, un aula (en principio), de estudio y aprendizaje, y una sala de profesores, de encuentro.

c) la representación semántica. Los niños y los adultos no son meros asistentes pasivos. Al contrario, reinterpretan la realidad en la que se mueven, pensándolo, analizándolo, recreándola 0, incluso, inventándola. Los seres humanos, en cuanto somos "objetos con mente", nos caracterizamos por nuestra capacidad de interpretación a partir de las propuestas físicas y funcionales del medio. En otras palabras, no manejamos únicamente los aspectos extensionales de la realidad, ni los funcionales, sino que gestionamos significados, a veces, impuestos por nuestra propia capacidad mental.

Cuando una persona entra en un espacio encuentra una propuesta formal que invita al uso, a disponer de él de acuerdo con su lógica. Así, la disposición de paredes, el color, la iluminación, temperatura, superficie, etc., crea diferentes ámbitos de actuación. Una variación en la organización de las características extensionales del espacio modifica la propuesta de actuación de las personas, que adecúan su comportamiento a la nueva disposición. En diseño arquitectónico, los estudios basados en el análisis de isovistas constatan, por ejemplo, que los patrones de interacción entre la personas varían según cambia la disposición de las estructuras móviles en el espacio (Scupelli, Kiesler y Fussel, 2007), alterando la dinámica del contacto ocular entre ellas.

La semántica del espacio hace referencia a los significados atribuidos a un contexto determinado. A cómo las personas interpretan el espacio, los objetos y las interacciones que ocurren en él (Tuulos, y Hämäläinen, 2017). Considerando una situación dada, podemos distinguir distintos tipos de representaciones: 
a) la representación formal del espacio;

b) la representación del uso del espacio;

c) la representación mental (subjetiva) del espacio.

Ambas representaciones no siempre coinciden. En numerosas ocasiones, la persona interpreta el espacio, simbolizando una situación superpuesta a él. Sin embargo, la interpretación semántica va más allá del análisis simbólico de las formas, los objetos y los espacios, para albergar contenidos metarrepresentados. Así, la representación del uso parece distanciarse con frecuencia de la representación subjetiva del uso.

\section{Relaciones entre espacio y aprendizaje}

La psicología del espacio educativo intenta dar respuestas a las condiciones más favorables para que alumnos y profesores puedan desarrollarse y aprender de forma óptima. La transformación semántica de un espacio puede posibilitar este efecto, contribuyendo a alinear los objetivos pedagógicos con los sentimientos, percepciones y disposiciones internas de los aprendices. Los espacios mal preparados, mal organizados 0, simplemente, nulos, desprovistos de toda capacidad para elicitar inquietudes, son focos de desmotivación, de falta de iniciativa, pues estimulan la pasividad, el reflejo de defensa y protección, si no de distancia y alejamiento.

La interacción entre aprendiz y espacio de aprendizaje no es casual pues, en el entorno de uso, se combinan constantemente experiencias pasadas con sensaciones actuales, creando y disponiendo de información sobre el entorno en tiempo real y de forma paralela a su actuación. Si la desviación entre expectativas y realidad física es grande, la disparidad producirá sensaciones negativas, algo que afecta al estado de ánimo y a la propia realización. Las condiciones de luz, temperatura y sonido pueden convertirse en predictores eficaces del rendimiento y su resultado posterior (Geen, McCown, y Broyles, 1985; Jennings, Nebs, y Brock, 1988; Weinstein, 1979). A ellas, se añadirán otros de carácter intrínseco, que proporcionan la construcción de la representación subjetiva. En esta investigación se analizan las variables de contexto ambiental y físico, en el proceso de interacción con la representación mental del espacio, para observar su impacto sobre las diferentes formas del uso que las personas hacen de los entornos que habitan.

\section{Método}

Diseño

La estructura de la investigación se basó en un diseño de tipo mixto paralelo y convergente. Para la recogida de información cualitativa se crearon dos soportes: un registro de identificación espontánea de espacios positivos, neutros y negativos, y un cuestionario semiestructurado, construido al efecto sobre plataforma online, con objeto de recabar datos sobre la interpretación subjetiva del espacio habitable (centro de estudios) de los participantes. Además, se configuraron tres grupos de enfoque, uno por curso académico, con el objetivo de contrastar y discutir los hallazgos categorizados principales del cuestionario. En el estudio cuantitativo se utilizaron medidas de calidad bioclimática (presión, temperatura, luz y sonido), junto con un registro de evaluación del espacio educativo, basado en un sistema de indicadores y criterios en forma de rúbricas.

\section{Participantes}

En el estudio participaron 260 personas. La selección se llevó a cabo siguiendo un procedimiento de muestreo 


\section{taphiya $\mathbf{4 6}$}

estratificado. La muestra elegida fue estadísticamente representativa de la población del Grado de Magisterio de la Facultad de Educación, y se distribuyó entre los estudiantes de primero, segundo y tercer cursos de Magisterio. Sus edades comprendían desde los 17;9 a los 32;3 (media, 18;4), Del total de la muestra, 201 participantes eran mujeres y el resto, hombres. Durante el tiempo de aplicación del diseño de investigación, un total de 17 personas fueron descartadas de la muestra, al no realizar todas las pruebas del procedimiento o no cumplimentar las escalas y registros en su totalidad. La muestra definitiva quedó en 243 personas (nivel de confianza 95\%; intervalo de confianza; error máximo admitido, 5\%).

Los grupos de discusión fueron constituidos siguiendo un procedimiento aleatorio por cada estrato, a partir de los estratos organizados en la constitución de la muestra.

\section{Instrumentos}

\section{Análisis semántico-transformacional.}

Fue creado un cuestionario electrónico que recogió información sobre juicios de preferencia del espacio en el centro de estudios. Para su realización se empleó el software Lime Sunvey, implementado sobre servidor Linux. Las cuestiones presentadas se dividieron en 4 categorías de estudio: Reconocimiento de las características del entorno, uso habitual de espacios, funcionalidad del espacio, e interpretación subjetiva del espacio. Las observaciones recogidas a través del instrumento fueron categorizadas y utilizadas en el análisis posterior, en los tres grupos de enfoque constituidos al efecto.

\section{Identificación de espacios transformables.}

Para la recogida de datos se creó un registro específico sobre identificación de espacios, con respuesta de elección libre. Contenía tres apartados, espacios positivos, espacios negativos y espacios neutros. Para cada uno de ellos se solicitaba un máximo de tres opciones.

\section{Grupos de discusión.}

Se constituyeron tres grupos de discusión de 6 participantes, elegidos al azar tras una propuesta general de candidatos. Cada grupo estuvo constituido por tres hombres y tres mujeres, todos estudiantes de la Facultad. A su vez, se estableció un grupo de discusión por cada uno de los tres segmentos de población, correspondientes a los tres primeros cursos académicos del estudio. Su objetivo fue contrastar los datos resultantes del análisis e interpretación del estudio, para observar información relativa a actitudes, sentimientos y preocupaciones de los participantes, no limitadas a las consideraciones previas del investigador. El tiempo de discusión prefijado fue de 90 minutos. La interacción fue grabada en audio y posteriormente transcrita.

\section{Medidas bioclimáticas.}

Para la recogida de datos ambientales se utilizó una estación meteorológica portátil (Netatmo ${ }^{\circledR}$ ). Se realizaron mediciones sobre los siguientes parámetros: temperatura, humedad relativa, presión, ruido ambiental y luminosidad, de todos los espacios elegidos tanto positiva, negativa o indiferentemente (espacios neutros). 


\section{Evaluación del espacio educativo.}

Para recoger los datos de evaluación de los espacios identificados, fue construida una rúbrica (ANEXO I) de evaluación a partir de una revisión y adaptación parcial de la escala ECERS.R (Harms, Clifford y Cryer, 1998). Se definieron cinco criterios de valoración, ambiente general, espacio físico, semántica, función (uso) e interacción. Cada criterio fue evaluado de acuerdo con la tabla de indicadores, entre 1 y 3 puntos.

\section{Procedimiento}

La aplicación del diseño de investigación se llevó a cabo en 5 fases que fueron conducidas a lo largo de tres meses, entre enero y abril. Durante las diferentes fases, los participantes fueron identificados mediante su n.i.a (número de identificación de alumno), de modo que fuese posible realizar un seguimiento individual de la participación.

Fase 1. Semántica de espacios. Los participantes en el estudio realizaron el cuestionario electrónico construido al efecto. Se recogieron datos sobre juicio de preferencia para espacios de aislamiento social, espacios sociales, espacios de recogimiento, trabajo en equipo y espacios de desagrado. Estos datos se compararon con los obtenidos en la fase de identificación de espacios para diseñar el mapa de representación semántica del espacio en el centro universitario y fueron utilizados como base para el tratamiento de grupos de discusión.

Fase 2. Identificación de espacios (libre elección). En la primera fase de la investigación, se solicitó, mediante el empleo del registro correspondiente, la libre elección de espacios a los participantes, mediante la siguiente instrucción tipo:

"Identifica tres espacios, por orden de prioridad, del (opción A: "más preferido"; B: "más desagradable"; (: "que no te diga nada") (1) al menos (según opción A, B o C) (3), en los que te sientes (mejor/peor/indiferente) en el Centro. Concrétalos lo máximo que puedas, y definelos con claridad para que nosotros podamos identificarlos como tú".

Fase 3. Variables bioclimáticas. A continuación, se tomaron medidas de las variables ambientales (temperatura, iluminación, nivel acústico y humedad relativa del aire), para los espacios positivos, negativos y neutros seleccionados por la muestra de estudio.

Fase 4. Los participantes evaluaron los espacios de uso. Para este propósito fue creada una rúbrica de evaluación (ver apéndice I), que contenía cinco criterios de evaluación. Impresión general del entorno, condiciones físicas y climáticas, semántica del espacio, uso (función) e interacción social.

Fase 5. Grupos de discusión. Los encuentros focales fueron llevados a cabo una vez recogidos los datos de las anteriores fases.

\section{Resultados}

Fase l. Análisis de campos semánticos asociados a la representación del espacio.

Se analizaron las respuestas dadas a través del cuestionario online. Para ello se siguió un procedimiento de análisis por claves semánticas, agrupando las valoraciones en cinco dimensiones: espacios de actividad individual (aislamiento), espacio de interacción social (socia), entornos de trabajo colaborativo y de equipo (trabajo equipo), espacios para la búsqueda de la interioridad (recogimiento) y espacios que producen una disconformidad (desagrado). 


\section{taphiya $\mathbf{4 6}$}

En la tabla 1 se muestran las asociaciones por campos semánticos realizadas por la muestra de participantes en esta fase de la investigación ( $N=243$ ), en preguntas de carácter abierto (libre expresión) incorporadas al cuestionario electrónico. Las diferencias en frecuencias absolutas se deben a los casos en que no existió respuesta o esta fue del tipo "no se me ocurre ninguno".

\begin{tabular}{|c|c|c|c|c|c|c|c|c|c|}
\hline \multicolumn{10}{|c|}{ TIPOLOGÍA SEMÁNTICA } \\
\hline Aislam. Social & & Social & & Trabajo & & Recogimiento & & Desagrad & \\
\hline CAF & 20 & CAF & 104 & CAF & 172 & CAF & 28 & - & - \\
\hline$B L B$ & 24 & - & - & BLB & 80 & BLB & 60 & BLB & 48 \\
\hline ENTR & 18 & ENTR & 48 & - & - & ENTR & 20 & - & - \\
\hline AULEXT & 4 & AULEXT & 24 & AULEXT & 12 & AULEXT & 5 & - & - \\
\hline BATH & 4 & - & - & - & - & BATH & 1 & - & - \\
\hline JARD & 9 & JARD & 40 & - & - & JARD & 72 & - & - \\
\hline - & - & ESCAL & 4 & - & - & - & - & - & - \\
\hline - & - & - & - & AULREF & 60 & AULAEF & 28 & AULREF & 68 \\
\hline - & - & - & - & - & & - & & & \\
\hline - & - & - & - & - & - & - & - & AUD & 4 \\
\hline - & - & - & - & - & - & - & - & SA & 12 \\
\hline- & - & - & - & - & - & - & - & HALLB & 4 \\
\hline - & - & - & - & - & - & - & - & SECRT & 8 \\
\hline
\end{tabular}

Tabla 1. Asignaciones por campos semánticos (frecuencias). Libre asociación. CAF= cafetería; BLB = biblioteca; ENTR = entrada (exterior); AULEXT: Aula exterior; BATH = baño; JARD =jardín; ESCAL = escalera; AULREF = Aula clase; AUD = auditorio; SA = salón de actos; HALLB = hall edificio B; SECRET = secretaría

De entre todos los espacios analizados, la cafetería del centro se considera tanto un espacio social (fr=104; 42,8\%) como un lugar de trabajo en equipo ( $\mathrm{fr}=172 ; 70,78 \%$ ), por encima, incluso, del potencial que tienen las propias aulas ( $\mathrm{fr}=60$; $24,69 \%$ o la biblioteca ( $f=80 ; 32,92 \%$ ), espacio este que dispone de lugares específicos para ello, sin embargo, poco reconocidos funcionalmente por los estudiantes. Un mayor ajuste ( $\mathrm{fr}=60 ; 24,69 \%)$ parece presentar el espacio central de la biblioteca, si bien cuenta con una tasa de desagrado alta (fr=48; 19,75\%). En suma, estos datos parecen mostrar la oportunidad de introducir mejoras en la organización de la biblioteca para ajustarla mejor al significado que dan los estudiantes, particularmente en lo relativo al trabajo en equipo. Por su parte, la asociación entre lugar de estudio y lugar de trabajo parece generar una representación negativa en los estudiantes respecto al aula (AULREF=68; 27,98\%, 
para desagrado), quienes prefieren los espacios más socializantes como la cafetería del centro, un espacio claramente polivalente. Se destaca, como argumento relevante, la menor tasa de elección de espacios para el aislamiento personal. La percepción del espacio, en general, como un entorno de relación invita a la reflexión sobre su uso y significado. Los estudiantes parecen buscar este tipo de contextos en los que se conjuga interacción social y aprendizaje por encima de aquellos que provocan el trabajo individual.

\section{Fase 2. Identificación de espacios. Juicios de preferencia.}

El estudio previo fue complementado con un registro abierto, en papel, de elecciones positivas, negativas y neutras, de los espacios del centro educativo. La libre elección añade validez a la recuperación intencional de espacios particularmente negativos o neutros, pues en este caso la decisión viene precedida por la representación mental (interna) del valor subjetivo (en la elección forzada, la decisión es precedida por la sugerencia externa del espacio).

En este caso se observa una mayor presencia de espacios desprovistos de significado (figura 1), algunos expresados de manera manifiesta (JARD, jardín, gradas, ESPENC y Hall entrada), a la vez que se incrementa gradualmente la tasa de respuestas vacías (NULL), entre las elecciones positivas $(n=18)$, negativas $(n=63)$ y de los espacios neutros $(n=261)$.

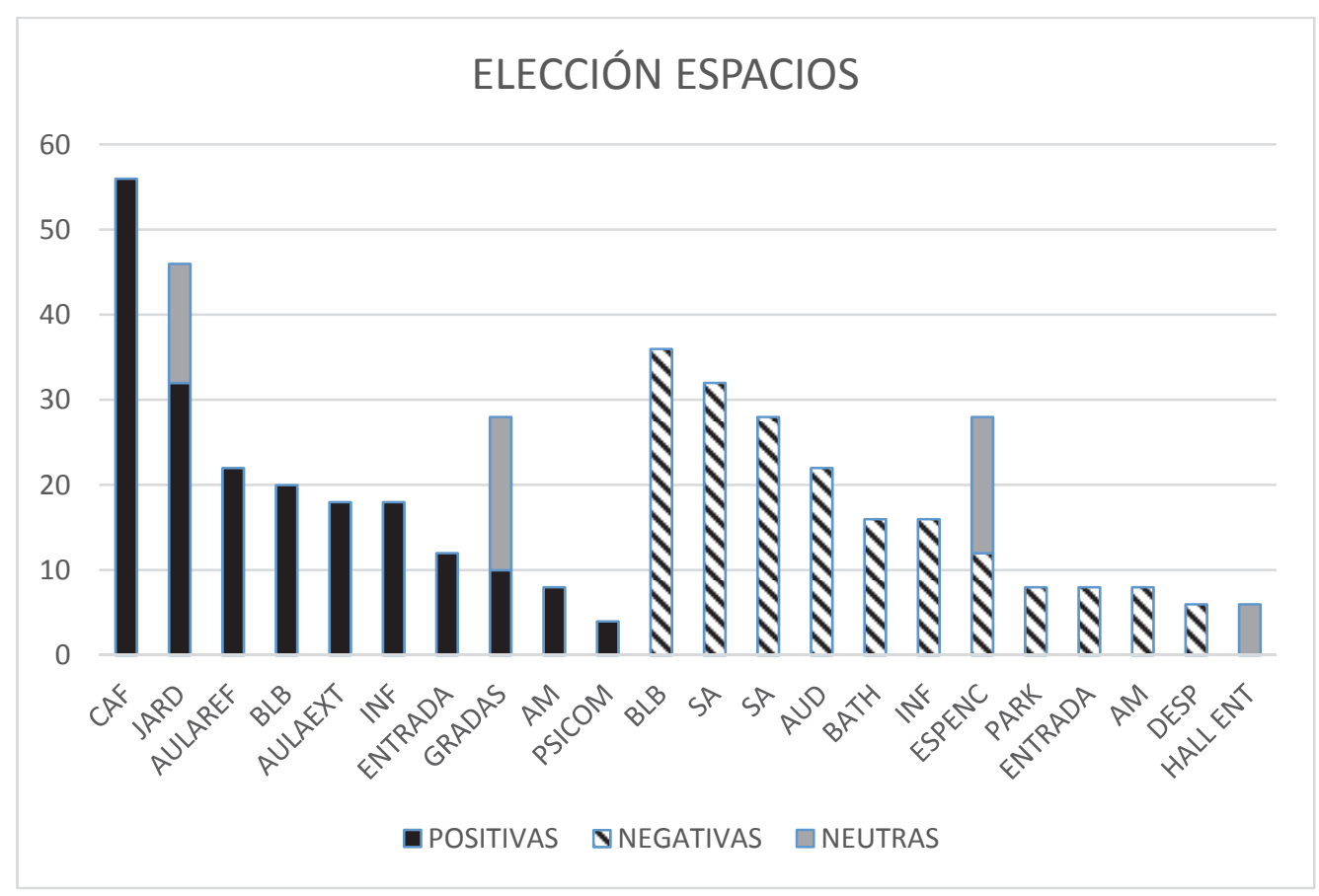

Figura 1. Identificación de espacios transformables (fase 1 de la investigación). Frecuencias acumuladas. Barras apiladas.

\section{Fase 3. Estudio bioclimático.}

Las medidas observadas no predijeron los resultados de la evaluación (tabla 2). Las medidas de condiciones de humedad, ruido, iluminación y temperatura, mostraron variaciones pequeñas durante el tiempo de uso. La temperatura se reguló mediante sistemas de calefacción general y estas diferencias observadas no mostraron un impacto directo sobre la evaluación de espacios por parte de los participantes. Este hecho también se aplicó a los 
espacios exteriores evaluados.

\begin{tabular}{|c|c|c|c|c|c|}
\hline \multicolumn{6}{|c|}{ ANOVA $^{\mathrm{a}}$} \\
\hline Modelo & $\begin{array}{r}\text { Suma de } \\
\text { cuadrados }\end{array}$ & $g \mid$ & Media cuadrática & $\mathrm{F}$ & Sig. \\
\hline Regresión & 4,572 & 4 & 1,143 & ,372 & $826^{b}$ \\
\hline Residuo & 61,458 & 20 & 3,073 & & \\
\hline Total & 66,029 & 24 & & & \\
\hline \multicolumn{6}{|c|}{ a. Variable dependiente: EVAL } \\
\hline
\end{tabular}

Tabla 2. Análisis de regresión. Influencia de las condiciones bioclimáticas sobre la evaluación de espacios.

Así pues, la discrepancia entre la evaluación positiva y las condiciones adversas de los espacios exteriores (jardines y entrada) se debe a factores ajenos a las condiciones bioclimáticas observadas. En la figura 2 se muestran algunas de estas relaciones.

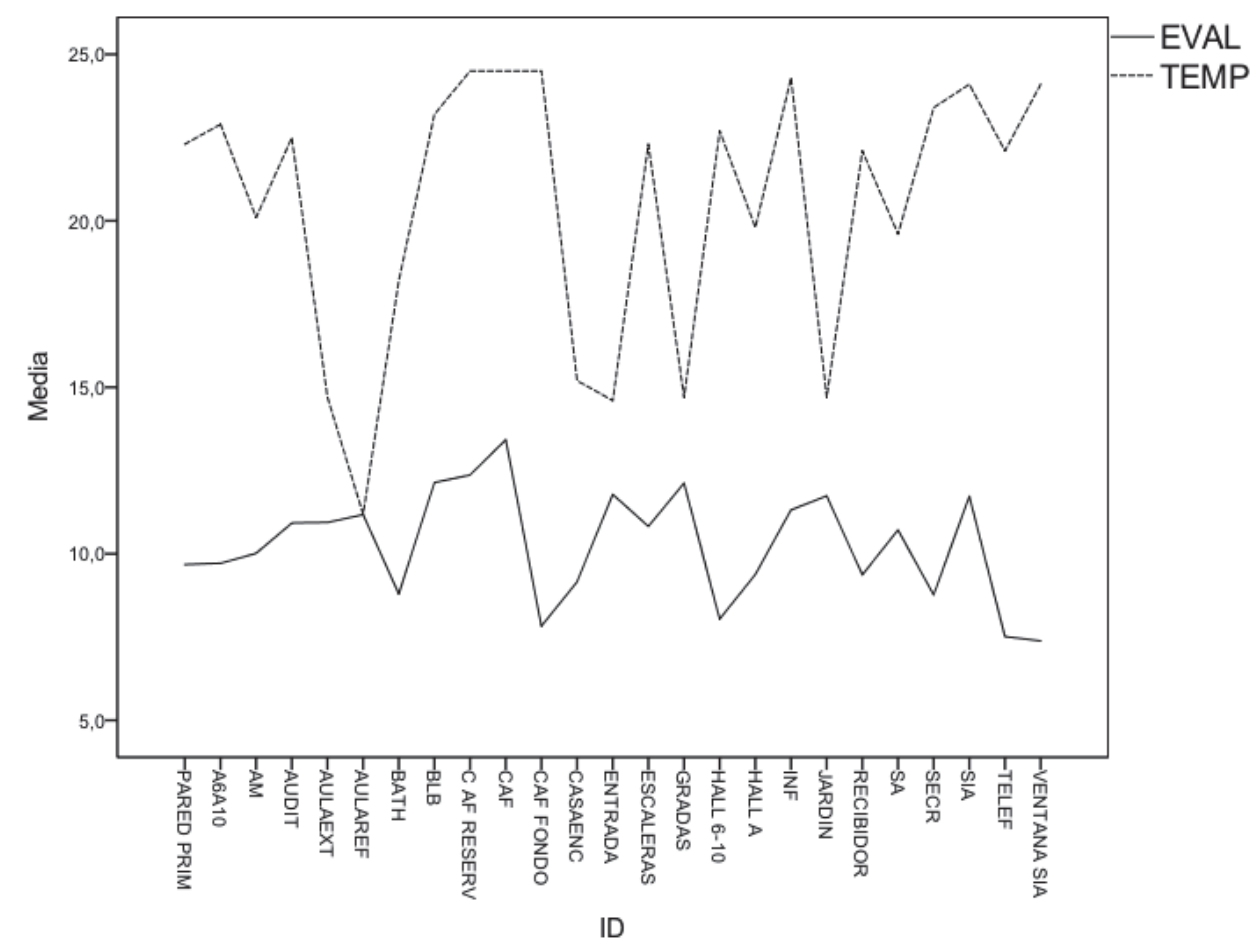

Figura 2. Relación entre condiciones de temperatura y grado de aceptación del espacio. Efectos principales. 
La temperatura promedio durante la recogida de datos (invierno) del espacio ajardinado fue baja (14,7 ${ }^{\circ}$ C; $44 \%$ humedad). Sin embargo, la apreciación en la evaluación forzada fue muy positiva (media 11,73). Las respuestas abiertas al cuestionario electrónico permitieron confirmar que los juicios de valor positivo se relacionaron con los aspectos estéticos y naturales del entorno y cómo estos propiciaban un espacio reconocido como muy positivo para el recogimiento personal (tabla 1). Estos marcadores de la representación subjetiva del espacio también parecieron manifestarse en relación con los espacios neutros. Así, para las mismas condiciones ambientales de la cafetería, reconocida muy positivamente como un espacio social y de interacción, algunas de sus localizaciones carecieron de interés para la mayoría (caso del espacio controlado del fondo de cafetería, con una discrepancia de 5 puntos (13-8) muy significativa. Por último, una tercera categoría de discrepancia vino marcada por las diferencias de uso. El espacio de atención a alumnos (SIA, con una valoración de 11,72 sobre 15 puntos posibles) fue considerado muy positivo al tiempo que el espacio que apenas distaba dos metros (Ventana SIA), fue valorado muy negativamente (7,38/15), pese a presentar las mismas condiciones bioclimáticas ( $24,1^{\circ} \mathrm{C}$ : 510 lúmenes; $41 \mathrm{db}$.; $39 \%$ humedad). Tomadas en conjunto, los resultados del estudio mostraron la presencia de tres variables que determinan la representación subjetiva en la valoración del espacio, la estética y naturaleza del entorno, el conocimiento del espacio y su uso.

\section{Fase 4. Análisis de varianza. Relación entre representación subjetiva y espacio funcional.}

A través de la rúbrica de evaluación se solicitó a los participantes su valoración en dos criterios diferenciados. Por un lado, el uso en relación con la función asignada al espacio y, por otro, la significación personal en relación con el uso que cada uno otorga al espacio habitado. En principio, ambas medidas deben coincidir pero puede darse el caso de que se produzcan discrepancias debidas a la representación subjetiva del espacio, motivo del análisis.

Se practicaron sendos análisis comparativos de medias, ANOVA de un factor (evaluación del espacio) X 2(semántico y funcional), intersujetos, para cada espacio identificado, para determinar si la hipótesis de igualdad de varianzas, entre la evaluación del criterio semántico y el criterio funcional y cada uno de los espacios asignados por el estudio. Los principales resultados se muestran en la tabla 3.

\begin{tabular}{|c|c|c|c|c|c|c|c|c|}
\hline & $\begin{array}{l}\text { Media } \\
\text { SEM }\end{array}$ & $\begin{array}{l}\text { Desv T. } \\
\text { SEM }\end{array}$ & $\begin{array}{l}\text { Media } \\
\text { FUNC }\end{array}$ & $\begin{array}{l}\text { ANOVA } \\
\text { Desv T. } \\
\text { FUNC }\end{array}$ & $\begin{array}{l}\text { Levene } \\
\text { (sig.) }\end{array}$ & $\begin{array}{l}\text { Media } \\
\text { cuadrática }\end{array}$ & F & $\begin{array}{l}\text { Sig. } \\
\text { (intergrup) }\end{array}$ \\
\hline AULREF & 2,138889 & 0,634802 & 2,375 & 0,567612 & 0,441822 & 2,007 & 5,535 & $0,017^{*}$ \\
\hline AULAEXT & 2,272727 & 1,969231 & 1,969231 & 0,8095 & 0,201 & 3,016 & 4,576 & $0,003^{*}$ \\
\hline JARDIN & 2,2222 & 0,791188 & 2,180556 & 0,8778 & 0,889109 & 0,063 & 0,09 & 0,765 \\
\hline ENTRADA & 2,2361 & 0,70197 & 2,2778 & 0,71645 & 0,902 & 0,063 & 0,124 & 0,725 \\
\hline BAÑOS & 1,8333 & 0,80491 & 2,0833 & 0,70711 & 0,031 & 2,25 & 3,92 & 0,050 \\
\hline BIBLIOT & 2,5417 & 0,60369 & 3,000 & 0,000 & 0,000 & 7,563 & 41,502 & $0,000^{*}$ \\
\hline SIA & 2,2361 & 0,61651 & 2,6111 & 0,51882 & 0,609 & 5,063 & 15,595 & $0,000^{*}$ \\
\hline SECRETARIA & 1,5278 & 0,76861 & 1,8472 & 0,83345 & 0,71 & 3,674 & 5,716 & $0,016^{*}$ \\
\hline AUDITORIO & 2,1231 & 0,81983 & 2,3846 & 0,62979 & 0,256 & 2,223 & 4,16 & $0,002^{*}$ \\
\hline CASAEXT & 1,9524 & 1,03482 & 1,7381 & 1,10563 & 0,547133 & 0,964 & 0,841 & 0,362 \\
\hline CAFETERIA & 2,6944 & 0,57259 & 2,7222 & 0,50969 & 0,429 & 0,028 & 0,095 & 0,759 \\
\hline
\end{tabular}




\section{taphiya $\mathbf{4 6}$}

\begin{tabular}{lrrrrrrrr}
\hline ESCALERAS & 2,0417 & 0,72067 & 2,2778 & 0,82602 & 0,111 & 2,007 & 3,34 & 0,070 \\
\hline INF & 2,3239 & 0,75166 & 2,4225 & 0,68997 & 0,669 & 0,345 & 0,663 & 0,417 \\
\hline AULA MUSICA & 2,0735 & 0,77875 & 2,0735 & 0,69789 & 0,32 & 0,000 & 0,000 & 1,000 \\
\hline GRADAS & 2,322 & 0,79742 & 2,2712 & 0,82693 & 0,976 & 0,076 & 0,116 & 0,734 \\
\hline SALON ACTOS & 2,1875 & 0,78973 & 2,4167 & 0,79448 & 0,886 & 1,26 & 2,009 & 0,16 \\
\hline HALLA & 1,8194 & 0,7185 & 1,8194 & 0,8278 & 0,203 & 0,000 & 0,000 & 1,000 \\
\hline PASILLOS & 1,7465 & 0,80566 & 1,8873 & 0,90316 & 0,505 & 0,704 & 0,962 & 0,328 \\
\hline RECEPCION & 1,7324 & 0,89375 & 1,8873 & 0,91884 & 0,945 & 0,852 & 1,037 & 0,31 \\
\hline
\end{tabular}

Tabla 3. ANOVA (unidireccional, por pares de variable). Cuadro resumen. Discrepancias entre representación subjetiva y funcional.

El análisis de varianza devolvió diferencias significativas para 6 de los espacios principales del Centro $(p<0,05)$. En estos casos, las discrepancias entre función física del espacio y semántica asociada mostraron la existencia de elementos personales que configuraban la representación subjetiva de los mismos, que pueden ser explicados en relación a los significados atribuidos por las personas en sus elecciones. Así, siguiendo la estructura semántica de la tabla 1, la disposición de un aula exterior en el jardín, diseñado inicialmente para dar una clase en la naturaleza, es un espacio que, distante de su función inicial (por ejemplo, la pizarra no se utiliza), se convierte en un espacio natural donde socializarse ( $\mathrm{N}=24$ ) y donde trabajar en equipo (N12). Aplicando un análisis similar, la biblioteca, que originalmente se concibe como un espacio de estudio e investigación, posee significados muy diferentes y repartidos entre las apreciaciones de los participantes para el estudio, exhibiendo incluso contrastes no fáciles de conciliar (por ej. trabajo en equipo, y $\mathrm{N}=80$, recogimiento, $\mathrm{N}=60$ ) que incluyen muestras de desagrado ( $\mathrm{N}=48)$, teniendo en cuenta que se trata de un espacio bien preparado y acondicionado como biblioteca y centro de recursos polivalente. En definitiva, la existencia de estas diferencias significativas puede orientar en la planificación y decisión sobre la intervención más eficaz para transformar el espacio educativo, mucho más allá de las cuestiones arquitectónicas, estéticas o funcionales.

\section{Fase 5. Grupos de discusión.}

Por último, el volcado y categorización de los datos extraídos de los grupos de discusión señaló algunas categorías de forma significativa a lo largo de los tres grupos de estudio. Así, por ejemplo, el sentimiento de "pertenencia del espacio" (el aula de referencia) influye en la apreciación positiva, independientemente de sus condiciones físicas (Luminosidad 0 Dimensiones). En ello también influye la organización del mismo (p. ej., la Organización del mobiliario). Otro dato importante relaciona las condiciones de representación subjetiva con la elección del espacio. Así, la biblioteca compartió protagonismo con la cafetería en la identificación de espacios donde se encuentra la tranquilidad. El primero, por las vistas hacia el entorno natural proyectado por los ventanales; el segundo, por el potencial socializador del contexto. En relación con la categoría Naturaleza, los jardines aparecen (junto con la Cafetería como espacio socializante) como un espacio proveedor de sensaciones positivas, por encima de los demás. Estos dos espacios provocaron trazas de intensidad del recuerdo en los participantes de manera preferente, señalando en la gran mayoría de los casos a factores de Socialización como facilitador de su anclaje en el recuerdo.

Se indagó de manera particular en los espacios que mostraron discrepancias en el análisis cuantitativo. El aula exterior, diseñada inicialmente con un propósito docente, presenta usos múltiples (por orden de Intensidad, Realización de Trabajos, Comida y Socialización), alejados de la función principal. Las diferencias observadas respecto al Servicio de Información parecen dirigirse a la amplitud del abanico de consultas (materiales, préstamos, encuadernación, consejos 
de diversa índole, inscripciones...), lo que lo convierte en un espacio multifuncional, que sobrepasa las características para la que fue creado inicialmente (información y atención académica). La biblioteca parece haber desplazado su función inicial (préstamo y consulta de libros), para ocupar otras funcionalidades representadas (estudio, trabajo, impresión de documentos, navegación por internet). Su uso aparece frecuentemente asociado a la representación de exámenes). Por último, la Secretaria apareció asociada a argumentos de las categorías Clima y Relación.

\section{Discusión}

Los principales resultados de esta investigación muestran la presencia de factores subjetivos en la representación del espacio que no se hallan directamente influidos por la variación bioclimática de las condiciones del entorno físico. Entre ellos se encuentran la estética del espacio, su relación con el entorno natural, el conocimiento del espacio por parte del observador y el uso subjetivo del espacio. Todos ellos, en conjunto, permiten realizar modelos de categorización semántica del espacio que refieren el mismo no como un mero entorno físico y arquitectónico, con una estructura dada y una función asignada, sino como un sistema inter e intrapersonal en el que la representación del espacio se sobrepone sobre la capa de representación formal. Esta dimensión psíquica del espacio añade una forma de comprenderlo que determina el modo en que puede ser transformado para mejorarlo, situando el foco de la transformación en la consecución de un mayor ajuste entre la propuesta formal del espacio y la representación subjetiva de las personas que lo habitan.

La investigación general ha puesto de manifiesto una influencia variable de las condiciones bioclimáticas sobre el proceso de aprendizaje que en ocasiones dependió del procedimiento de análisis utilizado. Gilavand, y Jamshidnezhad (2016) analizaron la influencia de fuentes de ruido en la motivación del alumnado de secundaria, encontrando diferencias significativas en la respuesta verbal de los participantes. Sin embargo, nuestros resultados, basados en medidas bioclimáticas, no resultaron coincidentes y estuvieron más en línea con otros estudios que mostraron que cuando la combinación de iluminación, sonido y temperatura se ajustan a la zona de confort, los resultados sobre el aprendizaje se normalizan, superando los ratios respecto a las zonas exteriores de confort (Marchand, Nardi, Reynolds, y Pamoukov, 2014), especialmente en tareas de escucha y comprensión. Las diferencias debidas al ambiente no son, por sí mismas, suficientes para explicar las diferencias en evaluación del espacio e incluso la actuación directa sobre algunos de estos factores (como la ventilación natural) es difíilmente viable (Mijakowski y Sowa, 2017). Las personas son conscientes de la influencia negativa de los excesos y defectos climáticos, si bien, en condiciones de ajuste normalizado, su capacidad predictiva sobre la variación del aprendizaje no parece ser significativamente determinante. Recientemente, Barret, Davies, Zhang y Barret (2015), han encontrado que el tamaño del efecto debido a la variación bioclimática (luz, temperatura, calidad del aire, pertenencia, flexibilidad, complejidad y color) es de un 16\% sobre el aprendizaje. Nuestros resultados coincidieron con esta observación respecto a la evaluación de los espacios de aprendizaje o de convivencia, lo que llevó a la consideración de factores adicionales de consideración subjetiva, en la representación del espacio. Junto con las cualidades ambientales, la organización del espacio refuerza la percepción de los estudiantes sobre su uso y eficacia. Los resultados muestran esta variable relacionada con los atributos espaciales del aula, particularmente la visibilidad y el mobiliario (Yang, Becerik-Gerber y Mino, 2013).

Los resultados obtenidos en este estudio añaden una visión diferente del tratamiento del espacio. La forma en que las personas utilizan el entorno se genera no solo a partir de la propuesta física y funcional sino también de la representación subjetiva, que genera una semántica particular del contexto. De esta manera, en todo entorno se presentan espacios de significado, positivos y negativos, y espacios neutros, en relación, respectivamente, al valor subjetivo 0 al conocimiento del mismo por parte del individuo. La investigación ha revelado algunas posibles variables en la construcción de la representación subjetiva. Tal es el caso, por ejemplo, del diseño de aula y su relación con la 
motivación y el compromiso de los alumnos (Appleton, Christenson, y Furlong, 2008). En el contexto de la psicología del espacio de aprendizaje, Granito y Santana (2016) han identificado, al igual que nosotros, como factor el valor que tanto profesores como especialmente los alumnos conceden al espacio en relación con el proceso de aprendizaje, señalándolo estos últimos por encima del impacto del profesor mismo. El reconocimiento del espacio se relaciona también con el desarrollo de la creatividad. Azeri, Parvizi, Khaleghi, y Hosseini (2015) han puesto de manifiesto la relación existente entre diseño y organización arquitectónico y variables psíquicas. La incorporación de elementos naturales, como plantas, espacios verdes, cuerpos celestes o uso del agua, junto con el empleo de formas suaves frente a agudas, de materiales naturales y presencia de entornos variados y flexibles, generan ambientes proactivos para el desarrollo de competencias creativas, motivadoras e innovadoras.

Existen modelos teóricos precedentes que intentan explicar las relaciones intrínsecas entre especio y comportamiento humano. Así, por ejemplo, el modelo E-B (Envirnoment-Behavior; Barret y Barrett, 2010), establece la existencia de tres principios facilitadores del aprendizaje, a saber, la disposición de entornos naturales (natura/ness), la individualización y el nivel de estimulación. En nuestro estudio hallamos que, además de la iluminación, el nivel acústico, la temperatura y calidad de aire, la presencia de espacios estéticos rodeados de naturaleza provoca evaluaciones positivas, incluso por encima de las anteriores en grado de intensidad. La representación subjetiva positiva del espacio parece ser un factor crítico que va más allá de la mera disposición de un entorno de confort (Barrett, Davies, Zhang, \& Barrett, 2016) para permitir la personalización individual del espacio y alcanzar el compromiso hacia el aprendizaje.

Un aspecto a resaltar en esta investigación es la relevancia que las personas otorgan a la representación general del espacio educativo y no solo a las aulas. Así, los espacios comunes, como la cafetería o los auditorios se convierten en espacios significativos, más allá del uso para el que fueron diseñados. La investigación general refuerza este hecho. Los espacios de tránsito (pasillos, corredores, senderos y conectores...) refuerzan el proceso de personalización del individuo, al facilitar la comunicación libre y sin restricciones (Tunner, 2009). Además, las aulas con amplios ventanales que miran a espacios naturales se muestran más accesibles a la representación social que aquellas que miran a espacios sin vida (aparcamientos o edificios neutros). En nuestro estudio, tanto los espacios ajardinados como las aulas de referencias analizadas arrojaron las tasas más altas de valoración positiva. Este hecho parece relacionarse con la posibilidad de restauración del equilibrio psicológico y fisiológico de las personas y su impacto sobre el bienestar y la salud individual (Hipp, Gulwadi, Alves, y Sequeira, 2015).

\section{Conclusiones. Limitaciones y futura investigación}

La representación subjetiva del espacio es un factor relevante para comprender la semántica asociada a los entornos educativos. La forma en que las personas se representan el espacio viene marcada por variables internas que se superponen a las físicas y bioclimáticas. Ambos niveles de representación no siempre son coincidentes y en ocasiones, los individuos otorgan significados diferentes a aquellos para los que fueron inicialmente diseñados. Las discrepancias observadas se deben a factores diversos, como el valor atribuido al espacio, su conocimiento, el modelo de interacción social, la estética asociada al espacio o la cercanía a la naturaleza. Estos rasgos afectan a la consideración del espacio por encima de la influencia de las condiciones bioclimáticas, particularmente cuando estas se encuentran moderadamente controladas. El ajuste de las discrepancias observadas entre ambos niveles de representación permite establecer pautas para el diseño arquitectónico y para la transformación eficaz del espacio educativo, orientada a la planificación de escenarios en los que las personas dialoguen con entornos cuyas propuestas formales se emparejen con la representación subjetiva que hacen de ellos. Este hecho, probablemente, incidirá no solo en los resultados académicos sino también en la mejora del clima individual y comunitario y, por tanto, en la salud y bienestar de la comunidad escolar.

\section{| 18}




\section{tapbiya $\mathbf{4 6}$}

Entre las limitaciones observadas se encuentra la dificultad para recabar datos objetivos a través del uso de cuestionarios electrónicos, algo que fue compensado, al menos parcialmente, con los registros de elección de espacios en papel. Si bien un gran número de estudios e investigaciones recientes utilizan este tipo de instrumentos, se hace preciso profundizar en la estructura psicológica de la representación del espacio educativo, mediante mecanismos y empleo de tecnología que permitan identificar la señal de preferencia de forma objetiva, alejando la decisión de la probabilidad del error debido a potenciales sesgos de respuesta verbal.

\section{Agradecimientos}

Esta investigación ha sido realizada gracias a la financiación aportada por el programa de ayudas a la investigación del CSEU La Salle-Madrid, en el proyecto asignado SPIEDU001. 


\section{taphiya $\mathbf{4 6}$}

\section{Bibliografía}

Appleton, J.J; Christenson, S.L. y Furlong, M.J. (2008). Student engagement with school: Critical conceptual and methodological issues of the construct. Psychology in the Schools, 45(5), 369-386.

https://doi.org/10.1002/pits.20303

AzerI, A.R.K.; ParvizI, R.; Khaleghi, S.J. y HosseinI, S.B. (2015). Effective Design Principles in Promotion of Children's Creativity in Residential Spaces. ASLI QoL2014 (Annual Serial Landmark International Conference on Quality of Life) / AQOL 2014 Istanbul (ABRA International Conference on Quality of Life), 26 - 28 December 2014, 202, 31-46. Istanbul, Turkey: Istanbul Technical University. https://doi.org/10.1016/j.sbspro.2015.08.206

BarRet, P.S. y BarRett, L. (2010). The potential of positive places: Senses, brain and spaces. Intelligent Buildings Internationa/, 2, 218-228. doi:10.3763/inbi.2010.0042

BARRetT, P.; DavieS, F.; ZHANG, Y. y BARRetT, L. (2015). The impact of classroom design on pupils' learning: Final results of a holistic, multi-level analysis. Building and Environment, 89, 118-133.

https://doi.org/10.1016/j.buildenv.2015.02.013

Barrett, P.; Davies, F.; Zhang, Y. y Barrett, L. (2016). The Holistic Impact of Classroom Spaces on Learning in Specific Subjects. Environment and Behavior, 13916516648735.

https://doi.org/10.1177/0013916516648735

BarRet, P. y Zhang, Y. (2009). Optimal Learning Spaces. Design Implications for Primary Schools. SCRI research report. Salford, UK: University of Salford.

Cleveland, B. y FISHER, K. (2014). The evaluation of physical learning environments: A critical review of the literature. Learning Environments Research, 17, 1-28. doi:10.1007/s10984-013-9149-3.

DavieS, D.; JINDAl-Snape, D.; Collier, C.; DigbY, R.; Hay, P. y Alan Howe, A. (2013). Creative learning environments in education-A systematic literature review. Thinking Skills and Creativity, 8, pp. 80-91, ISSN 1871-1871, http://dx.doi.org/10.1016/j.tsc.2012.07.004.

GEEN, R.G. MCCOWn, E.J. \& BRoyleS, J.W. (1985). Effects of Noise on Sensitivity of Introverts and Extraverts to Signals in a Vigilance Task, Personality and Individual Differences, 6, ( 2), pp. 237-241.

GIILVAND, A. YJAMSHIDNEZHAD, A. (2016). The Effect of Noise in Educational Institutions on Learning and Academic Achievement of Elementary Students in Ahvaz, South-West of Iran. International Journal of Pediatrics, 4(3), 1453-1463.

https://doi.org/10.22038/ijp.2016.6500

GISLASON, N. (2007). Placing education: the school as architectural space. Paideusis, 16(3), pp. 5.14.

Granito, V. y SAnTAnA, M. (2016). Psychology of Learning Spaces: Impact on Teaching and Learning. Journal of Learning Spaces, $5(1)$.

http://libjournal.uncg.edu/jls/article/view/882

HARMS, T.; ClIFFORD, D. y CRYER, D. (1998). ECERS-R, Early Childhood Environmental Rating-Scale. Revised version. New York, Teacher College Press.

HIPP, J. A.; GULWADI, G.B.; ALVES, S. y SEquelRA, S. (2015). The Relationship Between Perceived Greenness and Perceived Restorativeness of University Campuses and Student-Reported Quality of Life. Environment and Behavior, 48(10), 1292-1308.

https://doi.org/10.1177/0013916515598200 


\section{taphiya $\mathbf{4 6}$}

IMMS, W.; ClevelAnd, B. Y FISHER, K. (2016). Pursuing that elusive evidence about what Works in learning environment desing. En W. Imms, B Cleveland y K. Fisher (Eds). Evaluating Learning Environmets. Snapshots of Emerging Issues Methods and Knowledge. Rotterdam: Ense Publishers. Chapter1, pp. 3.20.

JenNingS, R.J.; NeBS, R. y Brock, K. (1988). Memory Retrieval in Noise and Psychophysiological Response in the Young and Old. Psychophysiology, 25, (6), pp. 633-644.

KenkMAnN, A. (2011). Adapting and designing spaces: children and their schools. CEPS Journal, 1(2), pp. 11-24 - URN: urn:nbn:de:0111-opus-60683

LACKNEY, J.A. (2008). Teacher environmental competence in elementary school environments. Children, Youth and Environments, 18(2), 133-159.

LAORDEN, C. y PéREZ, C. (2002). El espacio como elemento facilitador del aprendizaje: una experiencia en la formación inicial del profesorado. Pulso, 25. 133-146.

LeVEBVRE, H. (1991). The Production of Space. Oxford, UK: Blackwell

Marchand, G.C.; NardI, N.M.; ReynoldS, D. y Pamoukov, S. (2014). The impact of the classroom built environment on student perceptions and learning. Journal of Environmental Psychology, 40, 187-197.

https://doi.org/10.1016/j.jenvp.2014.06.009

MJJAKOWSKI, M. y SOWA, J. (2017). An attempt to improve indoor environment by installing humidity-sensitive air inlets in a naturally ventilated kindergarten building. Building and Environment, 111, 180-191. https://doi.org/10.1016/j.buildenv.2016.11.013

PARK, E.I. y CHOI, B.K. (2014). Transformation of classroom spaces: traditional versus active learning clasroom in colleges. The International Journal of Higuer Education Research, 68(5), pp., 749-771.

PerolinI, P.S. (2011). Interior Spaces and the Layers of Meaning. Design Principles and Practices: An International Journal, 5(6), pp. 166-174.

POLDMA, T. (2010). Transforming interior spaces: Enriching subjective experiences through design research. Journal of Research Practice, 6(2), Article M13.

http://jrp.icaap.org/index.php/jrp/article/view/198/199

SCUPELLI, P.G.; KIESLER, S. Y FusSELL, S.R. (2007). Using isovist views to study placement of large displays in natural settings. En CHI '07 Extended Abstracts on Human Factors in Computing Systems (CHI EA '07). New York, NY, USA: ACM. 2645-2650. D0l=http://dx.doi.org/10.1145/1240866.1241056

TANner, C.K. (2009) Effects of school design on student outcomes. Journal of Educational Administration. Vol. 47 (3), pp.381 - 399

TUULOS, T. Y HÄMäLÄIINEN, M. (2017). Bridging physical and social space: Practices and behavior in co-creation platforms. En T.A Björklund, M. Laakso, S. Kirjavainen y K. Ekman (eds.). Passion-based co-creation. Helsinki; Aalto University. ISBN 978-952-60-3740-0, pp. 120-131.

WeINSTEIN, C. S. (1979). The Physical Environment of School: A Review of the Research. Review of Educational Research, 49, (4) pp. 577-610.

YANG, Z;; BECERIK-GeRBER, B. y MinO, L. (2013). A study on student perceptions of higher education classrooms: Impact of classroom attributes on student satisfaction and performance. Building and Environment, 70, 171-188. https://doi.org/10.1016/j.buildenv.2013.08.030 


\title{
taphiya $\mathbf{4 6}$
}

\section{Resumen.}

La presente investigación ha tenido como objetivo analizar la representación subjetiva del espacio educativo. La hipótesis fundamental plantea la existencia de discrepancias ocasionales entre esta y la representación funcional del entorno. Para analizar los resultados se planteó un diseño de tipo mixto, convergente paralelo, basado en el uso combinado de cuestionarios y registros semiestructurados, para el análisis cualitativo, y toma de medidas bioclimáticas y uso de rúbricas en la valoración de espacios como instrumentos para el análisis cuantitativo. Nuestros resultados mostraron que las condiciones ambientales ejercieron un efecto no predictivo sobre la evaluación del espacio y, sin embargo, fueron constatadas discrepancias significativas entre la representación subjetiva del espacio (semántica asociada) y su función. Cruzando los datos de análisis, se concluye la existencia de variables subjetivas que caracterizan la representación, como son la estética del entorno, el carácter natural, el conocimiento del espacio o el modo de interacción social. Las conclusiones de la investigación aportan valor acerca de la identificación eficaz de prioridades de transformación que aproximen la funcionalidad a la forma en que las personas utilizan y reconocen el espacio que habitan.

Palabras clave. Semántica del espacio; Diseño; Transformación; Representación Subjetiva; Espacio Educativo.

\begin{abstract}
.
The present research has the purpose of analyzing the subjective representation of educational spaces. Its main hypothesis poses the issue of the existence of eventual discrepancies between that and the functional representation of the environment. Results were analyzed by building a mixed quasi experimental design, based on both questionnaires and records, bioclimatic measures and inferencial statistics applied to space assessment by using rubrics. To analyze the results, a mixed-type parallel convergent design was proposed, based on the combined use of questionnaires and semistructured registers, for qualitative analysis, and taking of bioclimatic measures and the use of rubrics in the assessment of spaces as instruments for quantitative data. Our results showed that environmental conditions exerted a non predictive effect over the assessment of space. However, there were outstanding significant discrepancies between the subjective representation of space (its associated semantycs) and its assigned function. Crossing data analysis, we conclude the existence of subjetive variables that caracterize the mental representation, as the aesthetics of the environment, its natural character, the knowledge about space or the way to interact with others. Research conclusions provide value about the effective identification of changemaking priorities that approximate functionality to the way in which people use and recognize the space in which they live.
\end{abstract}

Key-words. Semantyc of Space; Design; Changemaking; Subjective Representation; Educative Space.

Javier Herrero-Martín

Centro Superior de Estudios Universitarios La Salle

j.herrero@lasallecampus.es 